\title{
Les expositions professionnelles associées à l'utilisation des appareils portatifs de radiographie industrielle gamma en France*
}

\author{
J. CANDIOTTI ** \\ (Manuscrit reçu le 5 février 1986)
}

RÉSUMÉ

\begin{abstract}
On parle beaucoup des expositions accidentelles en radiographie gamma. Certains organismes nationaux et internationaux en ont recherché les raisons sans trop les approfondir. Une étude plus poussée menée par l'auteur fait ressortir des causes propres à la radiographie gamma. On peut alors être étonné qu'il n'y ait pas eu plus d'incidents. La mise en place d'une réglementation adaptée à la profession et la prise de conscience par les opérateurs des risques qu'ils peuvent faire encourir, surtout à des tierces personnes, font maintenant que cette activité peut ne pas être aussi dangereuse que certains le prétendent. Une action doit, toutefois, être entreprise dans la formation du personnel qui laisse encore à désirer pour l'aide et la personne compétente.
\end{abstract}

\section{ABSTRACT}

Accidental overexposures of industrial radiographers are topics of the day. Their causes have been investigated by several national and international bodies. In a more thorough study, the author emphasizes the causes specific of industrial radiography, and one can be surprised that incidents should not occur more often. Owing to regulations adapted to the profession and the operators'awareness of the risks to which they can expose others, this activity should not be so hazardous. However, actions should be undertaken in personnel training, particularly as concerns the ancillary personnel and the qualified individual.

Aux cours des dernières décennies, l'utilisation des radioéléments artificiels dans l'industrie s'est beaucoup développée. De nombreux domaines d'activité ont été complètement transformés et de nombreux débouchés sont apparus. Ainsi, dans le domaine du contrôle non destructif, la radiographie gamma joue un rôle prépondérant et figure parmi les six techniques officiellement reconnues ${ }^{(1)}$. Chacune de ces techniques a un champ d'ap-

* Communication présentée au congrès franco-italien "Les expositions professionnelles - aspects méthodologiques et expériences pratiques", organisé à Nice du 29 au 31 janvier 1986 par la Société française de radioprotection et l'Associazione italiana di protezione contro le radiazioni.

* Commissariat à l'énergie atomique, Institut de protection et de sûreté nucléaire, Département d'analyse de sûreté, BP 6, 92265 Fontenay-aux-Roses Cedex.

(1) A savoir : radiographie $x$ et gamma, ultrasons, magnétoscopie, ressuage, courants de Foucault et étanchéité. 
plication limité de sorte qu'elles sont plus complémentaires les unes des autres que concurrentes.

On classe les appareils de radiographie gamma en trois grandes catégories basées sur le mode d'éjection de la source et en fonction de leur mobilité. Actuellement, il s'avère que plus de $90 \%$ des appareils sont portatifs et comportent l'éjection du porte-source hors de l'appareil. Pour les autres appareils, s'ils sont mobiles, leur nombre est très restreint et s'ils sont fixes, ils sont placés dans des installations bien protégées et surveillées. Le véritable problème qui se pose donc est avant tout celui des appareils portatifs contenant des sources d'iridium 192 de 100 curies maximum.

En 1977, le Comité scientifique des Nations Unies [5] avait en quelques lignes, situé le problème concernant les causes d'accidents survenus avec de tels appareils. II écrivait : "En radiographie industrielle, on utilise des sources importantes, exposant à de fortes doses dans un court laps de temps, en général dans de mauvaises conditions et avec un minimum de mesures directes de radioprotection".

La même année, dans son rapport annuel, la Nuclear regulatory commission (NRC) des Etats-Unis [1] s'inquiétait des incidents survenus et essayait d'en comprendre les raisons.

"Un incident arrive lorsque, soit par négligence, soit par une mauvaise application des règles, soit du fait de l'équipement, la source radioactive n'est pas entièrement revenue de sa position d'utilisation à sa position de stockage et l'opérateur ne s'en est pas aperçu avant de s'approcher de l'appareil".

Donc, depuis 1977 , le problème était posé. En s'appuyant sur ces deux déclarations, il était déjà possible de le cerner.

En 1978, la parution d'un rapport du National council on radiation protection and measurements américain (NCPR) $|4|$ relatif à l'enseignement en radiographie gamma, apporte encore des éléments nouveaux. Ce document consacre un chapitre entier aux causes d'accidents. II en cite sept principales à savoir:

1. présence de rayonnement de forte énergie,

2. forte activité de la source,

3. présence de personnes sur le chantier à proximité des zones surveillées,

4. défauts de l'appareil,

5. télécommande de l'appareil dans une zone interdisant le contrôle visuel de la zone à surveiller,

6. mauvaise interprétation des règlements,

7. formation de l'opérateur déficiente.

Les trois premiers facteurs $(1,2,3)$ dépendent des essais techniques qui sont exigés et des lieux où ils doivent être effectués. Des rayonnements de forte énergie peuvent être nécessaires pour contrôler les pièces métalliques épaisses. Une forte activité peut être exigée pour atteindre des temps d'exposition économiquement acceptables. Les trois derniers facteurs $(5$, $6,7)$ sont dus à des actions humaines. L'étude des incidents montre que la plus grande majorité d'entre eux est imputable à une défaillance de l'opérateur plutôt qu'à un défaut de l'appareil (4e facteur). Toutefois, ces accidents 
auraient souvent pu être évités par l'utilisation d'appareils plus sûrs, par exemple, conformes au récent décret 85-968 d'août 1985. [2]

Ces différentes approches pour rechercher les causes des accidents sont-elles suffisantes? L'expérience montre qu'elles ont encore besoin d'un complément car elles n'expliquent pas tout.

La question posée est de savoir pourquoi la radiographie industrielle gamma serait-elle plus dangereuse que les autres activités utilisant des radioéléments artificiels? On ne peut pas se contenter de généralités qui pourraient être appliquées à n'importe quel appareil d'irradiation gamma.

Une étude plus approfondie a donc été menée. Elle a porté principalement sur la conception et l'utilisation des appareils, la formation du personnel et les incidents connus. De nombreux enseignements en ont été tirés. II n'est pas possible de les citer tous et c'est volontairement qu'il n'est pas question ici de la conception des appareils, sujet suffisamment important pour être traité à part. Les enseignements figurant ci-dessous sont très spécifiques et méritaient d'être connus.

$\left.1^{\circ}\right)$ Un appareil portatif de radiographie gamma remplit trois fonctions : - il sert de conteneur de stockage lorsque la source n'est pas utilisée ; - il devient projecteur lorsqu'il est utilisé sur un chantier ;

- il est transformé en emballage de type B (U) pour le transport de la source sur la voie publique.

Ces fonctions multiples, d'une part, font que de nombreux organismes officiels sont concernés et d'autre part, représentent une somme de risques potentiels non négligeables. En effet, ce n'est pas parce qu'un accident a eu lieu sur un chantier qu'il faille se désintéresser de la partie transport ou stockage et vice versa. Tout accident doit faire l'objet d'une enquête approfondie afin d'en étudier les répercussions éventuelles qu'il aura sur l'ensemble des sûretés. L'accident de Montpellier, par exemple, bien que survenu à l'intérieur d'une usine a montré que l'appareil n'était plus un emballage de type B (U).

$2^{\circ}$ ) Par rapport au parc des appareils d'irradiation gamma, celui des appareils de radiographie gamma est relativement élevé. On en compte 1200 environ en France, dont 200 seraient transportés journellement sur les routes. Dans le cadre de la réglementation du transport des matières radioactives (classe 7 ), une réglementation particulière leur est appliquée. Les quelques incidents routiers (quatre en sept ans) ont montré que la fonction emballage avait bien joué son rôle.

$\left.3^{\circ}\right)$ L'appareil portatif de radiographie gamma ne se trouve pas dans une installation fixe bien protégée et surveillée, mais sur des chantiers créés à la demande suivant les besoins. Cet appareil est peu encombrant, autonome dans son fonctionnement, donc très mobile. On ne saura jamais à l'avance où l'accident se produira. Sa grande souplesse d'emploi permet de l'utiliser partout, même dans des endroits les plus inattendus.

Sur un chantier, bien souvent il n'est pas possible d'établir un écran de protection efficace et, comme le temps d'exposition est imposé par l'épaisseur de la pièce à radiographier, il ne reste plus à l'opérateur que la ressource de compter sur la distance qui, elle-même, est limitée par la longueur du câble de télécommande. Ce sont les raisons pour lesquelles, sur 
un chantier, les opérateurs reçoivent des doses plus fortes que ceux qui travaillent en installation fixe.

$\left.4^{\circ}\right)$ Pour qu'un appareil soit rentable, il doit être utilisé au maximum. II n'est pas rare qu'avec un tel appareil, un opérateur effectue plus de 20000 éjections de sources par an. Comparativement aux autres appareils d'irradiation gamma, la probabilité de créer un accident en radiographie gamma est donc plus grande.

$5^{\circ}$ ) Sur un chantier, l'opérateur est, pour ainsi dire, livré à lui-même. L'aide qui doit obligatoirement l'accompagner n'a généralement aucune formation. II n'a donc personne derrière lui pour l'empêcher de commettre le geste irrémédiable. Aussi, bien souvent, pour honorer son contrat, il aura tendance à intervenir en cas d'incident matériel. II créera donc un deuxième incident plus grave par ignorance, en voulant remédier à un incident matériel, au départ sans conséquence. Cette notion de deuxième incident ne doit pas être perdue de vue, car il arrive que des équipes d'intervention l'ont provoqué par méconnaissance des systèmes de sécurité de l'appareil qui ne sont pas les mêmes d'un type d'appareil à un autre.

Pour revenir au problème de l'aide, on a constaté qu'il a été la cause de la plupart des incidents. En effet, l'opérateur pour gagner du temps demande à son aide d'effectuer à sa place certaines opérations. Dans un proche avenir, le rôle de l'aide devra être revu. Certaines grandes entreprises exigent maintenant auprès des sociétés prestataires de services que l'aide soit en possession du CAMARI (1) comme l'opérateur. Cette solution de facilité ne paraît pas avoir fait l'unanimité. En effet, certains préconisent une formation élémentaire qui serait à définir.

$\left.6^{\circ}\right)$ Sur un chantier, l'opérateur, en plus de son travail technique, doit également assurer la protection des travailleurs, du plublic et de l'environnement. II assume donc des responsabilités non négligeables. II doit pouvoir, sans ambiguïté, appliquer les consignes et pouvoir, par l'autorité qu'elles lui donnent, obliger les personnes réticentes ou trop envahissantes à se plier à ses injonctions. II ne doit pas se laisser intimider par des personnes qui seraient susceptibles de lui en imposer à divers titres.

L'opérateur effectue généralement son travail de nuit, bien souvent sans tenir compte des conditions météorologiques et de la nature du terrain. II doit donc être encore apte physiquement en fin de travail, tard dans la nuit, pour procéder à tous les contrôles avant de déposer son appareil dans le local de stockage.

$\left.7^{\circ}\right)$ Dans certains cas, pour n'avoir pas respecté les consignes de sécurité, des opérateurs ont été la cause d'irradiation très forte de tierces personnes, le plus souvent par la perte de la source pour ce qui concerne les accidents les plus graves. Les appareils en cause, jusqu'ici couramment utilisés, ne possédaient pas de dispositifs sûrs pour éviter cette perte.

On peut se demander, à la lecture de toutes ces causes, comment par des actions simples, on peut remédier à des incidents. En fait, il faut agir sur quatre facteurs: la source radioactive elle-même, la formation du personnel, l'appareil, les enseignements tirés des incidents.

(1) CAMARI : Certificat d'aptitude à manipuler les appareils de radiologie industrielle. 


\section{a) La source radioactive elle-même}

Il s'agira de définir la source à utiliser en fonction de l'épaisseur de la pièce à radiographier et de définir, ensuite, l'activité qui sera la plus rentable sans toutefois irradier le personnel et les alentours.

\section{b) Formation du personnel}

Comme l'a écrit en 1965 M. Chassany de la Cogéma, Marcoule, "La radiographie gamma est un métier et comme tout métier, elle s'apprend et doit être pratiquée uniquement par du personnel qualifié" [6].

On parle trop souvent de l'opérateur ; or, la réglementation s'adresse à trois catégories de personnes : l'employeur, la personne compétente et les travailleurs.

Chacun, en ce qui le concerne, a un rôle à jouer. Dans une entreprise, la sûreté dépendra des connaissances de l'employeur en la matière et de l'aptitude de la personne compétente. L'attitude de l'opérateur, bien qu'il soit titulaire du CAMARI, dépendra de la qualification de la personne compétente et du choix fait par l'employeur pour la désigner. Quant à l'aide, comme nous l'avons vu plus haut, sa formation laisse à désirer. Tout est à faire.

L'employeur doit connaître les risques qu'il prend en assumant une telle responsabilité. S'il connaissait tous les règlements, bien souvent, il ne se lancerait pas dans une telle aventure. La sûreté n'est pas à la portée de tous et ne pas la respecter peut être grave de conséquences.

Une cause actuelle d'accident, qui n'a pas été citée faute de preuves tangibles, pourrait provenir de la concurrence déloyale qui s'est instaurée depuis la crise économique, concurrence entre sociétés et concurrence entre opérateurs de sociétés différentes sur un même chantier.

\section{c) L'appareil}

II a été beaucoup demandé aux constructeurs depuis l'accident de Montpellier (révision des normes AFNOR et décret). Plus un appareil est simple dans sa conception, moins il sera cause de défaillance. Actuellement, vouloir en demander plus en sophistiquant davantage l'appareil, n'atteindrait pas le but recherché, à moins de repartir à zéro et de concevoir un nouveau type d'appareil. Quel est le constructeur, dans la conjoncture actuelle, qui prendrait un tel risque?

De plus, un tel appareil ne pèse que $15 \mathrm{~kg}$ environ. Plus il sera léger, plus il aura de chance de se vendre. Aussi, quelques millimètres gagnés sur le diamètre du porte-source, ce sont quelques kilogrammes en moins pour l'appareil. La France, par rapport aux autres pays, a divisé par 2 le débit de dose au contact de l'appareil portatif ( $1 \mathrm{mGy} / \mathrm{h}$ au lieu de 2 ou $100 \mathrm{mrad} / \mathrm{h}$ au lieu de 200). Diminuer encore cette norme obligerait le constructeur, soit à augmenter la protection radiologique, donc le poids, ce qui est impensable, soit à diminuer l'activité de la source, ce qui est également impensable. La période de l'iridium 192 étant courte, ce débit de dose au contact est rarement atteint, ce qui est une sauvegarde pour l'opérateur. Le problème n'aurait pas été le même avec une source de cobalt 60 de période beaucoup plus longue.

\section{d) Enseignements tirés des incidents}

C'est un des points capitaux de la sûreté. La connaissance des incidents est essentielle. Ce qui peut être considéré comme un cas insignifiant 
pour une entreprise, ne laissera pas indifférent les organismes de sûreté lorsque ce cas se répétera plusieurs fois ailleurs. Tout incident devrait être signalé, or, par peur de sanctions administratives, les entreprise se taisent. II n'y a rien de pire que la loi du silence. II est regrettable, qu'actuellement en France comme dans la plupart des pays étrangers, les incidents ou accidents ne fassent pas systématiquement l'objet d'une enquête technique suivie d'une publication dans une revue spécialisée. Les quelques incidents connus l'ont été soit parce qu'ils ont paru dans la presse, avide de tels événements, soit parce qu'ils ont fait l'objet d'une communication d'ordre médical.

Par contre, aux Etats-Unis, tout incident est signalé et fait l'objet d'une enquête. En 1982, par exemple, la NRC dans son document NUREG/BR0024 [3] relatif à la sécurité du travail en radiographie gamma donne une statistique très intéressante des accidents dus à la manipulation de sources radioactives, survenus aux Etats-Unis entre 1971 et 1980 , soit sur une période de 10 ans. On s'aperçoit que pour les très nombreux incidents ayant entraîné de très faibles irradiations de personnes, la radiographie gamma n'est pas en cause. Par contre, pour les quelques cas de très fortes irradiations de personnes, la radiographie gamma vient en tête.

II est un fait qu'une source radioactive sortie de son appareil présente des dangers indéniables. Or, c'est en radiographie gamma qu'on en trouve le plus et partout. Toutefois, grâce à l'action entreprise par les pouvoirs publics, le nombre d'accidents graves est très limité par rapport au très grand nombre d'éjections de source.

\section{CONCLUSION}

Certaines statistiques montreraient qu'actuellement il y aurait plus d'opérateurs irradiés qu'il y a 10 ans par exemple. On pourrait alors penser que toutes les dispositions prises récemment l'auraient été pour rien. Or, en approfondissant le problème, on s'aperçoit que cette augmentation du nombre d'opérateurs irradiés provient de l'aboutissement d'une campagne d'information entreprise ces dernières années en même temps que la mise en place d'une réglementation adaptée à la profession. Les opérateurs, grâce au CAMARI, ont pris conscience du danger qu'ils encourent et qu'ils font encourir aux autres. Ils savent que toute erreur de manipulation peut engendrer de graves conséquences et c'est la raison pour laquelle ils n'hésitent plus à consulter leur médecin du travail. Les incidents de radiographie n'ont pas augmenté tout d'un coup. Nous voyons apparaître un phénomène nouveau qui est très encourageant. Ce que certains camouflaient, il y a quelques années, n'est plus possible maintenant. Une collaboration encore plus étroite entre le médecin et le technicien est indispensable si l'on veut améliorer la formation des opérateurs en supprimant ces manipulations intempestives encore trop nombreuses.

De plus, la question posée est de savoir si le risque encouru par les opérateurs ou que ceux-ci font courir à des tierces personnes vaut la peine d'être pris ? L'expérience a montré qu'il était actuellement difficile de se passer des services rendus par la radiographie gamma dans le domaine du contrôle non destructif. Contester son utilité parce qu'il y a possibilité d'incidents, mettrait en cause tous les progrès réalisés ces dernières 
années grâce à une technique qui a très largement fait ses preuves. II n'y a pas, à ma connaissance, d'activités qui ne présentent aucun risque et le monde continue à vivre. Cette nouvelle activité doit être acceptée et pour cela les organismes de sûreté s'y emploient. En prenant donc les précautions qui s'imposent, en améliorant celles qui existent, en donnant au personnel une formation de haut niveau, en exigeant du constructeur un appareil présentant un maximum de sûreté sans aller au-delà, la radiographie gamma, bien qu'elle ne soit pas à la portée de tous, peut ne pas présenter les dangers que certains craignent. On aura beau réglementer, automatiser, robotiser, le dernier geste restera toujours à l'homme qui devra, à tous moments connaître la position de sa source et s'assurer, en fin de travail, que celle-ci est bien en position de stockage et en lieu sûr.

On trouvera en annexe, la description de quelques cas d'incidents caractéristiques dont certains auraient pu avoir de graves conséquences si le personnel n'avait pas été compétent.

\section{ANNEXE}

\section{RELEVE DE QUELQUES INCIDENTS CARACTERISTIQUES}

1०) Défaillance du matériel : le constructeur n'a pas procédé à un contrôle des pièces avant la sortie d'usine

En décembre 1978, au cours d'une opération de radiographie dans une compagnie aérienne, le porte-source s'est désolidarisé de la télécommande et est resté coincé dans la gaine d'éjection. Il a fallu faire appel à une équipe d'intervention pour récupérer la source.

L'enquête a montré que la goupille qui tient solidaire les deux parties constituant le porte-source n'avait pas été mise. Les trous prévus à cet effet n'avaient pas été percés en usine.

\section{$2^{\circ}$ ) Erreur de l'opérateur qui fait découvrir un défaut de fabrication du câble de télécommande}

En 1980, un opérateur a réussi à brancher la gaine de télécommande en oubliant de raccorder au préalable le câble de télécommande au porte-source. Cette opération est, d'après le constructeur, techniquement impossible. Le porte-source poussé par le câble est resté coincé dans la gaine d'éjection. A chaque retour du câble de télécommande, on entendait les sécurités se remettre en place, ce qui pouvait laisser penser que le porte-source était rentré en position de stockage puisque, en fonctionnement normal, c'est lui qui actionne ces sécurités en revenant à cette position.

L'enquête a montré que le câble de télécommande possédait à son extrémité un boulet de plomb de même diamètre que la tête du porte-source, et c'est ce boulet qui déclenchait les sécurités de façon intempestive. Un accident grave a pu être évité grâce au contrôle de rentrée de source effectué chaque fois à l'aide d'un radiamètre comme le prescrit la réglementation en vigueur. Une équipe d'intervention a pu récupérer la source sans irradiation de personne.

\section{3०) Tierce personne irradiée par la faute de l'opérateur}

Un opérateur, en fin de travail, transporte en voiture son appareil pour le stocker dans le local prévu à cet effet. Au cours du transport, le porte-source tombe sur la route à l'intérieur de l'usine. Le lendemain matin, un ouvrier le ramasse et le garde dans sa poche toute la journée. Le soir, l'opérateur s'aperçoit de la disparition du porte-source et donne l'alarme. Le porte-source est retrouvé au vestiaire dans la combinaison de l'ouvrier qui l'avait ramassé. 
L'enquête a montré la mauvaise conception de cet appareil : languette de retenue du porte-source défectueuse, verrouillage de l'obturateur inopérant, bouchon de protection de l'appareil côté éjection perdu, déplacement de l'obturateur en position travail.

Cet accident a été favorisé par l'adjonction d'un ressort et d'un fourreau pour rendre l'appareil conforme à la norme. Création d'une sécurité négative : le ressort poussant le porte-source vers l'extérieur au lieu de le retenir.

4) Erreur de l'opérateur. L'équipe d'intervention, par méconnaissance du fonctionnement des dispositifs de sécurité de l'appareil, crée un deuxième incident

En 1980, un opérateur réussit à brancher la gaine de télécommande sans avoir raccordé le porte-source au câble. Le porte-source poussé par le câble est resté coincé dans la gaine. L'équipe d'intervention ignorant le fonctionnement des dispositifs de sécurité de l'appareil a eu des difficultés pour récupérer la source. Un membre de l'équipe a été légèrement exposé. Ce deuxième incident aurait pu être facilement évité si l'équipe d'intervention avait connu au préalable le fonctionnement de cet appareil.

\section{5०) Nombreuses fautes commises par la société ; l'incident était inévitable}

En 1982, une société effectuant des radiographies gamma sur un chantier a procédé à 8 ou 9 mises en place de films radio en présence de la source sortie, alors que celle-ci aurait dû être chaque fois en position de stockage, obturateur fermé. C'est en démontant l'appareil en fin de travail que l'opérateur s'aperçut que le portesource s'était désolidarisé du câble de télécommande et était resté coincé dans la gaine d'éjection.

Causes de l'incident : utilisation d'un câble de télécommande détérioré ; son raccordement au porte-source, effectué par l'aide, n'a pas été vérifié par l'opérateur ; pas de contrôle de rentrée de source en position stockage.

\section{L'enquête a fait ressortir de nombreuses fautes commises par la Société :}

- comptabilité des sources et des appareils non tenue de façon régulière,

- pas de fiche de travail en milieu radioactif,

- confusion dans la distribution des films dosimètres ; l'aide nouvellement embauché prend le film d'un opérateur démissionnaire,

- le responsable de la société n'a pas averti le SPR de l'incident et a essayé d'y remédier lui-même, créant un deuxième incident,

- le magasinier savait que le câble était défectueux et s'était trompé de casier ;

et a montré que l'opérateur n'avait pas respecté les consignes de radioprotection. En effet :

- il a laissé opérer son aide qui ne possédait aucune qualification,

- il a laissé son appareil de mesure dans sa voiture, donc n'a pas pu contrôler la rentrée de la source en position de stockage,

- il a laissé son détecteur d'appoint à alarme sonore sur arrêt,

- il n'a pas vérifié régulièrement son stylo dosimètre à lecture directe.

$\left.6^{\circ}\right)$ Action de la personne compétente qui évite de créer un deuxième incident

Lors d'une manipulation sur une tour de refroidissement à $40 \mathrm{~m}$ de haut, l'opérateur au cours d'un déplacement laisse tomber son appareil qui chute de $10 \mathrm{~m}$. II le récupère et monte à $60 \mathrm{~m}$ de haut. En voulant alors vérifier son appareil avant de s'en servir, le porte-source est projeté hors de l'appareil et tombe sur le caillebotis percé de trous. La personne compétente avertie aurait pu se servir d'une pince à distance pour remettre le porte-source dans l'appareil. Elle a eu peur d'être maladroite et de faire tomber le porte-source, alors en position d'équilibre instable, ce qui aurait provoqué un incident assez grave étant donné le chantier au bas de la tour. La personne compétente divisa l'opération en trois, se gardant pour lui la partie la plus délicate. Les trois personnes n'ont pris que de très faibles doses. 
Cause de l'incident :

Lors de la chute de $10 \mathrm{~m}$, l'appareil ne possédant pas ses bouchons de protection a chuté sur l'arrière du porte-source détériorant la languette de retenue déjà réputée pour être défectueuse sur ce type d'appareil. L'opérateur aurait dû avoir le réflexe de vérifier de suite le bon état de marche de son appareil, sans attendre de se trouver à $60 \mathrm{~m}$ de haut.

\section{REFERENCES}

[1] ETATS-UNIS. Nuclear regulatory commission (NRC). Annual report 1977. NUREG-0400.

12| FRANCE. Travail, emploi et formation professionnelle (Ministère). Décret n० 85-968 du 27 août 1985 modifiant l'article R. $233-83$ du code du travail et définissant les conditions d'hygiène et de sécurité auxquelles doivent satisfaire les appareils de radiographie industrielle utilisant le rayonnement gamma. J.O.R.F., 14 sept. 1985, 10587-10589.

[3| MCGUIRE S.A., PEABODY C.A. Working safety in gamma radiography, a training manual for industrial radiographers. NUREG/BR-0024, 1982.

[4] NATIONAL COUNCIL ON RADIATION PROTECTION AND MEASUREMENTS (NCRP). Radiation safety training criteria for industrial radiography, NCRP report N.61. Washington: NCRP, 1978.

[5| NATIONS UNIES. Comité scientifique pour l'étude des effets des rayonnements ionisants. Sources et effets des rayonnements ionisants. New York : Nations unies, 1977.

|6| RODIER J., CHASSANY M. Incidents caractéristiques survenus à Marcoule au cours de gammagraphies industrielles. In : Congrès international sur la radioprotection dans l'utilisation des radioéléments, Paris, 13-15 décembre 1965. Le Vésinet: SCPRI, 1967, 21-23. 